\title{
Adquisición de codas líquidas en una muestra de niños del español de Chile*
}

\author{
Pilar Andrea Vivar Vivar* \\ Eduardo Arteaga Viveros ${ }^{* * *}$ \\ Leslie Elizabeth Figueroa Alarcón ${ }^{* * * *}$
}

\begin{abstract}
Resumen
Se investigó la adquisición de las codas liquidas /1/ y / / / en posición medial de palabra en una muestra compuesta por 161 niños hablantes del español de Chile, divididos en cuatro grupos etarios desde los 2,0 a los 3,11 años. La producción de las codas fue analizada como correcta, sustituida y omitida. Se observó que el rendimiento de estas estructuras aumenta en la medida que avanza la edad, al igual que la disminución del fenómeno de omisión. La sustitución se mantiene relativamente estable en todos los grupos etarios analizados, mientras que se observan patrones de sustitución relacionados con el punto de articulación y la escala de sonancia.
\end{abstract}

Palabras Clave: adquisición fonológica, codas mediales liquidas, español de Chile.

\section{Liquid coda acquisition in a sample of Chilean Spanish speaking kids}

\begin{abstract}
This research is about the adquisition of the liquid codas /l/ and / $/$ in medial position within a sample of 161 participants, who are speakers of Chilean Spanish. Those were divided in four age groups from 2,o up to 3,11 years old. The coda production was analysed as correct, substituted and ommitted. It has been observed that the output improves in higher ages, as well as dimishes the omission at the same time. The substitution is maintained relatively stable in all the age groups we have analysed, while at the same time we observe substitution patterns related to the place articulation and the sonority hierarchy.
\end{abstract}

Key Words: phonological acquisition, liquid medial position codas, Chilean Spanish.

Recibido: 05/07/2018

Aceptado: 07/o1/2019

Este artículo se inscribe en el Proyecto Fondecyt de Iniciación $N^{\circ} 11140414$ : “Desarrollo fonológicofonético infantil en niños entre 2,0 y 3,11 años. Un estudio transversal - descriptivo con aplicación de la prueba articulatoria CEFI". Investigadora principal Dra. Pilar Vivar V.

** Chilena. Doctora en Lingüística por la Universidad de Concepción. Académica en Universidad Católica de Temuco, Temuco, Chile.pvivar@uct.cl

*** Chileno. Magíster en Desarrollo Humano Local y Regional por la Universidad de la Frontera. Sociólogo en Universidad Católica de Temuco, Temuco, Chile. earteaga@uct.cl

*** Chilena. Profesora de Lengua Castellana y Comunicación por la Universidad Católica de Temuco, Temuco, Chile.lfigueroa2014@alu.uct.cl 


\section{Introducción}

Durante los primeros estadios de adquisición, son las codas consonánticas una de las estructuras fonológicas que presentan mayor dificultad para los niños, lo cual se manifiesta en estrategias de omisión y sustitución durante la producción de las mismas. El estudio y análisis de estas estructuras silábicas durante la adquisición del lenguaje ha sido abordado desde distintas perspectivas teóricas y metodológicas como, por ejemplo, aspectos prosódicos, posición de la coda dentro de la palabra, frecuencia de la coda en la lengua objeto, escala de sonancia y el tipo de segmento que compone la coda.

En este último aspecto, algunos autores han observado que para lenguas como el inglés, el catalán y el español, los niños en sus primeras etapas producen segmentos semiconsonánticos ([w, j]) en posición de coda (Borràs-Comes \& Prieto, 2013; Morales-Front, 2006; Oropeza Escobar, 2001). Específicamente, para el español, variados estudios, además, han evidenciado que para la posición de coda aparecen primero las nasales y al final las vibrantes (Fernández López, 2009; Goldstein \& Cintrón, 2001; Núñez-Cedeño, 2007, Polo, 2018). Para el español de Chile no existen hasta ahora descripciones específicas sobre la adquisición de las codas consonánticas, aunque se podría hipotetizar que los rasgos distintivos de los segmentos que en español pueden ocupar la posición de coda, influyen en el orden de adquisición de las mismas. Uno de los objetivos principales de esta investigación se relaciona con el aspecto anterior, ya que se analizó la emergencia de las codas compuestas por segmentos líquidos los que se han considerado como de adquisición tardía en la mayoría de la literatura en español. Se analizaron las siguientes codas': /ls/, /ld/, /lt/, /rb/, /rs/, /rt/ y /rm/ en posición medial de palabra. Se entrevistaron a 161 niños/as entre 2,0 a 3,11 años y la muestra se dividió en 4 grupos etarios. Para la recolección de los datos se utilizó el Cuestionario para la Evaluación de la Fonología Infantil (CEFI) en su versión adaptada a la variante del español chileno (Vivar y León 2009; Vivar 2009). El análisis se basó en la contabilización de las articulaciones correctas, sustituidas y omitidas según la variable edad y un análisis de tipo cualitativo en el que se comparan los tipos de sustituciones y omisiones para cada tipo de coda.

También llamados grupos heterosilábicos. 


\section{Marco Teórico}

\subsection{Adquisición de los grupos consonánticos}

Tradicionalmente, la sílaba se ha definido como un conjunto de segmentos agrupados en torno a un núcleo, la vocal. Para referirse a las posiciones o grupos de segmentos dentro de la sílaba se utilizan los términos ataque, núcleo, coda y rima. El ataque es la consonante o grupo de consonantes que precede al núcleo dentro de la sílaba, mientras que la coda es lo que sigue al núcleo. El núcleo y la coda se agrupan en una unidad mayor denominada rima (Harris, 1991). En relación con la frecuencia de los tipos silábicos en español, (Quilis, 1999) basándose en recuentos realizados sobre la lengua hablada presenta las siguientes frecuencias relativas para el español:

Tabla $\mathbf{N}^{\circ} 1$. Frecuencias de estructuras silábicas en español.

\begin{tabular}{|c|c|c|c|}
\hline 1. & $\mathrm{CV}$ & $\ldots$ & 55,81 \\
\hline 2. & CVC & ......................... & 21,61 \\
\hline 3 . & V & $\ldots$ & 9,91 \\
\hline 4. & VC & $\ldots$ & 8,39 \\
\hline 5. & $\mathrm{CCV}$ & …................ & 3,14 \\
\hline 6. & CCVC & $\ldots$ & 0,98 \\
\hline 7. & VCC & .................... & 0,13 \\
\hline 8. & CVCC & $\ldots$ & 0,02 \\
\hline 9. & CCVCC & ................... & 0,01 \\
\hline
\end{tabular}

De acuerdo con la Tabla $\mathrm{N}^{\circ} 1$, la estructura silábica $\mathrm{CV}$ es la más frecuente $(55,81 \%)$, mientras que, las rimas complejas son menos frecuentes $(21,61 \%)$ y generalmente se forman con una sola consonante (sol, pan). Muy pocas veces $(0.13 \%)$ son rimas formadas por dos consonantes en la posición palabra-media (constante).

Específicamente en el caso de la coda en español, en esta pueden aparecer consonantes oclusivas, fricativas, líquidas y nasales en posición media de palabra, mientras que en posición final de palabra solo son posibles $/ \mathrm{n} /, / \mathrm{s} /, / \mathrm{e} /, / \mathrm{l} /, / \mathrm{r} /$ junto a $/ \mathrm{d} /$, que se neutraliza, $\mathrm{y} / \mathrm{x} /$, que tiene una frecuencia muy baja (Bosch, 2004). Con respecto a la frecuencia de aparición la coda más frecuente es fricativa /s/, seguida de nasal /n/, lateral /l/, y finalmente la vibrante / / / (Polo, 2018). En relación a la 
complejidad fonotáctica, el español no permite más de una consonante en la posición final de palabra, excepto en los casos de palabras extranjeras como película [‘film]; por su parte, en posición medial de palabra sí pueden aparecer las codas complejas, como por ejemplo /ns/ o /rs/ como en perspicaz o construcción (Borràs-Comes y Prieto, 2013)

Desde la perspectiva de la adquisición fonológica, existen evidencias empíricas que comprueban el rol de la sílaba como una unidad lingüística crucial en el desarrollo de la gramática infantil. Por ejemplo, Jusczyk (1997), en su investigación sobre la percepción del habla, cuenta de la preferencia de los bebés por analizar el flujo continuo del habla sobre la base de la sílaba como unidad. En la producción del habla, durante el balbuceo, MacNeilage \& Davis (1990) observan que los bebés son más propensos a experimentar con nuevos segmentos en el contexto de estructuras silábicas estables. Posteriormente, durante el período de adquisición de las estructuras silábicas son los grupos consonánticos (GC) las estructuras fonológicas que presentan mayor dificultad para los niños, lo cual se manifiesta en algunas estrategias como la omisión, reducción y sustitución de dichas estructuras; no obstante, a pesar de existir patrones universales de adquisición entre distintas lenguas (marcadez), las características fonotácticas y la frecuencia de aparición de fonemas y tipos silábicos en cada lengua juegan un rol crucial en el momento en que los niños optan por una estrategia u otra.

\subsection{Adquisición de codas consonánticas}

En general, se considera que la adquisición de las codas silábicas corresponde a una adquisición tardía en la cual los niños/as, además de ampliar y complejizar su repertorio silábico, deberán incluir en estas nuevas estructuras los diversos fonos que corresponden a ellas (Fikkert, 1994; Freitas, 1997). En este proceso, la adquisición de las codas resulta ser un proceso complejo para los niños, porque las sílabas con codas son fonológicamente más complejas, poseen más restricciones fonotácticas y además son menos marcadas que los ataques silábicos (Polo, 2018). A pesar de que los estudios en torno a la adquisición y desarrollo de las codas silábicas son escasos en español, las investigaciones se han enfocado principalmente en aspectos como la posición de la sílaba dentro de la palabra (intermedia o final de palabra), acentuación (acentuada o inacentuada), los tipos de codas y la frecuencia de aparición de las codas en una lengua (Polo, 2018). 
Respecto al orden en la adquisición de las codas según su posición dentro de la palabra, se ha evidenciado que las codas en final de palabra (CV.CVC) se adquieren primero que las de posición intermedia (CVC.CV) en holandés (Fikkert, 1994), francés (Rose, 2000), inglés (Kirk \& Demuth, 2006) y catalán (Borras-Comes \& Prieto, 2013; Prieto, 2006). Sin embargo, para el español no existe consenso, pues algunos autores plantean que los niños adquieren primero las codas de final de palabra (Oropeza Escobar, 2001), mientras que otros, señalan que primero se adquieren las codas intermedias (Lleó, 2003), y por último, algunos autores no han hallado diferencias en la adquisición de las codas según la posición en la que se encuentren (Núñez-Cedeño, 2007).

Para el español, según Oropeza Escobar (2001) las secuencias heterosilábicas son las primeras en ser adquiridas, es decir, aquellas formadas por la coda de la primera sílaba y el ataque de la sílaba consecutiva. Según la autora, específicamente, las codas se adquieren progresivamente: 1 . la primera secuencia consonántica es la conformada por nasal más consonante homorgánica, 2. se incorpora la lateral [1] y después [r], 3. aparece la obstruyente [s], aunque suele sustituirse primero por [h] y luego, por la continua no estridente $[\Delta]$, 4. se adquieren las oclusivas en coda silábica. Según la autora, el comportamiento de algunos segmentos consonánticos solo es explicable atendiendo simultáneamente tanto a la posición que ocupan como a las características fonéticas específicas del segmento contiguo. En los grupos heterosilábicos en los que /s/ es el primer elemento (coda en interior de palabra), se sustituye por [h], luego de que ha sido elidido por algún tiempo. Este elemento sustituto mantiene los rasgos +consonántico y -sonoro, pero pierde la característica supraglotal, en otras palabras, la relacionada con el punto de articulación y modo de articulación. Por ejemplo: “espejo” - [ehpéxo], “casco” - [káhko], “fantasma” - [fantáhma], "cisne" - [qíhne]. En los grupos homosilábicos el segmento líquido del grupo es elidido en las primeras etapas del desarrollo fonológico. Posteriormente es sustituido por [y] que es un segmento a la vez -silábico y -consonántico y comparte con las líquidas el rasgo +aproximante. Por ejemplo: "plato" - [pyáto], “chicle” - [èíkye], "rifle” - [dífye], "crema” [kyéma], "estrella" - [ehtyéja]. Siguiendo la hipótesis de la importancia de las características prosódicas durante la adquisición de las codas, Lleó (2003) estudió el desarrollo de las codas en el habla de dos niños monolingües madrileños, cuyas edades promedio rondaban los 2,3 años. 
Su hipótesis indica que en el desarrollo de las codas intervienen tanto la prosodia como la semántica. Los resultados de su estudio demuestran que las codas son producidas solo cuando la sílaba es la acentuada o marcada. Esto quiere decir que en español, las codas en posición intermedia de palabra se adquieren primero que las de final de palabra, a diferencia del inglés (Goad \& Brannen, 2003) y el francés (Rose, 2000).

Polo (2018) analizó la producción de 8517 codas de dos niños entre 1,7 y 2,7 años hablantes de español. Los niños menores a 2 años produjeron más y precisas codas en sílabas acentuadas, específicamente en posición final de palabra. Con más de 2 años de edad los niños produjeron las codas en posición intermedia acentuada, mientras que sustituyeron los fonos en las sílabas inacentuadas y en posición final de palabra. Polo (2018) menciona que el desarrollo de las codas se relaciona con la estructura de la sílaba, cuando los niños ya han desarrollado esta última se enfocan en la calidad segmentaria, en español los niños seguirían las predicciones de marcadez. La sustitución de las codas es un fenómeno independiente a la estructura silábica, por ejemplo: las codas sonoras se sustituyen por otra sonora y las codas obstruyentes se sustituyen con otra obstruyente.

En resumen, la coda ha llamado la atención de muchos de los que investigan la adquisición. Sostienen que su desarrollo responde a una variabilidad considerable en la que intervienen aspectos como la longitud de de las palabras y la ubicación de la acentuación (marcadez) en la palabra (Kirk \& Demuth, 2006; Prieto \& Bosch-Baliarda, 2006), la posición de la articulación (Song, Demuth \& Shattuck-Hufnagel, 2012), tipos de consonantess (Kehoe \& Stoel-Gammon, 2001 Stites, Demuth \& Kirk, 2004), frecuencia léxica (Stoel-Gammon, 1998), probabilidad fonotáctica (Zamuner, Gerken \& Hammond, 2004), las características de frecuencia específicas de las codas (Zamuner, Gerken \& Hammond, 2005) y, las consideraciones/restricciones fonéticas (Fey \& Gandour, 1982).

\section{Metodología}

Se abordó un diseño metodológico mixto, cualitativo y cuantitativo, no experimental y de tipo transeccional descriptivo. Las unidades de observación en la investigación correspondieron a participantes entre 2,0 a 3,11 años. La muestra se dividió en 4 grupos etarios: grupo etario I (2,0 a 2,5 años), grupo etario II (2,6 a 2,11 años), grupo etario III (3,0 a 3,5 
años) y grupo etario IV (3,6 a 3,11años). En definitiva, se entrevistaron a 192 participantes de los cuales se hicieron válidas para el análisis 161 encuestas. Para la recolección de los datos se utilizó el Cuestionario para la Evaluación de la Fonología Infantil (CEFI) en su versión adaptada a la variante del español chileno (Vivar y León 2009; Vivar 2009). El cuestionario CEFI compuesto por 49 láminas, de las cuales para los objetivos de este estudio se seleccionaron aquellas láminas que contenían palabras con codas líquidas:

Tabla $\mathbf{N}^{\circ}$ 2. Codas líquidas.

\begin{tabular}{|c|c|c|c|c|c|}
\hline $\begin{array}{c}\text { Coda }+ \\
\text { consonante }\end{array}$ & & Palabra & $\begin{array}{c}\text { Coda }+ \\
\text { consonante }\end{array}$ & & Palabra \\
\hline$/ \mathrm{l}+\mathrm{c} /$ & $\begin{array}{l}/ \mathrm{ls} / \\
/ \mathrm{ld} / \\
/ \mathrm{lt} /\end{array}$ & $\begin{array}{c}\text { bolsa } \\
\text { falda } \\
\text { alto }\end{array}$ & $/ \mathrm{f}+\mathrm{c} /$ & $\begin{array}{l}/ \mathrm{rb} / \\
/ \mathrm{rs} / \\
/ \mathrm{rt} / \\
/ \mathrm{rm} /\end{array}$ & $\begin{array}{c}\text { árbol } \\
\text { cárcel } \\
\text { torta } \\
\text { enfermo }\end{array}$ \\
\hline
\end{tabular}

La evaluación se aplicó de manera individual procurando un ambiente acústicamente adecuado y sin factores distractores. La prueba se presentó como un juego en el cual se les solicitó a los informantes que expresen verbalmente lo que veían en cada lámina. Sus respuestas fueron inducidas por las entrevistadoras con preguntas del tipo: ¿qué es eso?, ¿qué hace?, ¿de qué color es?, etc. y cada entrevista fue grabada en un equipo TASCAM D-40. En relación con el análisis de los datos, esta investigación se basó en una perspectiva articulatoria que permite identificar y describir cómo se van insertando fonemas y grupos consonánticos en el sistema fonológico infantil, por lo que la unidad de análisis fue el elemento lingüístico, es decir, la emisión de una consonante o grupo consonántico. Con respecto a la metodología en particular, con la finalidad de elaborar patrones evolutivos, se contabilizó el número de emisiones correctas para cada fonema y se obtuvo el promedio de articulación normal de estas unidades lingüísticas en cada grupo etario. Además, se contabilizó la frecuencia de sustituciones fonológicas y fonéticas para cada fonema y grupo fonemático, lo que permitió observar las rutas articulatorias frecuentes que utilizan los niños durante el proceso de adquisición fonológica. El procedimiento anterior permitió observar y sistematizar de manera apropiada el proceso de inserción y ejecución de elementos lingüísticos al sistema fonológico infantil en cuanto a desarrollo lingüístico. 


\section{Resultados}

Se presentan los resultados según articulación correcta de las codas / / / y /l/, así como también, los porcentajes sustitución y omisión para los mismos segmentos. Los resultados son comparados según los grupos etarios.

\subsection{Articulaciones correctas, sustituidas y omitidas}

El Gráfico $\mathrm{N}^{\circ} 1$ presenta los resultados generales de las emisiones infantiles en relación con las emisiones correctas, sustituidas y omitidas para las codas consonánticas / / / y /l/. En general, se evidenció que la articulación correcta de /l/ es mayor que la de / $/$ /. En este sentido, "la coda vibrante /l/ se emitió correctamente 293 veces de un total de 805 emisiones posibles $(36,4 \%)$; en contraste, la coda lateral / $\mathrm{r} /$ se emitió correctamente 235 veces de un total de 1127 (21\%). En resumen: se observa que la coda vibrante /1/ tiene un porcentaje de emisión correcta de un $36,4 \%$, a diferencia de la coda lateral $/ \mathrm{f} /$ que obtuvo un 21\%. En relación con las sustituciones, se evidenció que la coda /l/ se sustituyó 108 veces (13,4\%) de las 805 veces que se pudo sustituir, mientras que la coda / / / se sustituyó 281 veces (25\%) de las 1127 veces que pudo ser sustituida. De acuerdo a estos datos, se observa que el porcentaje de sustitución de la coda vibrante $/ \mathrm{f}$ / es mayor que el de la coda lateral /1/. Con respecto a las omisiones se observó que el porcentaje de omisión de la coda / $/$ / es mayor que el de la coda /l/, siendo un $46 \%$ para la primera y un $40 \%$ para la segunda. La coda líquida vibrante / / / se omitió 518 veces de un total de 1127 que pudo ser omitida, mientras que la coda líquida lateral /l/ se omitió 321 veces de las 805 veces que pudo se omitida.

Gráfico $\mathbf{N}^{\circ}$ 1. Porcentaje articulación correcta, sustituida y omitida para las codas / $/ \mathrm{l} / \mathrm{y} / \mathrm{l} /$.

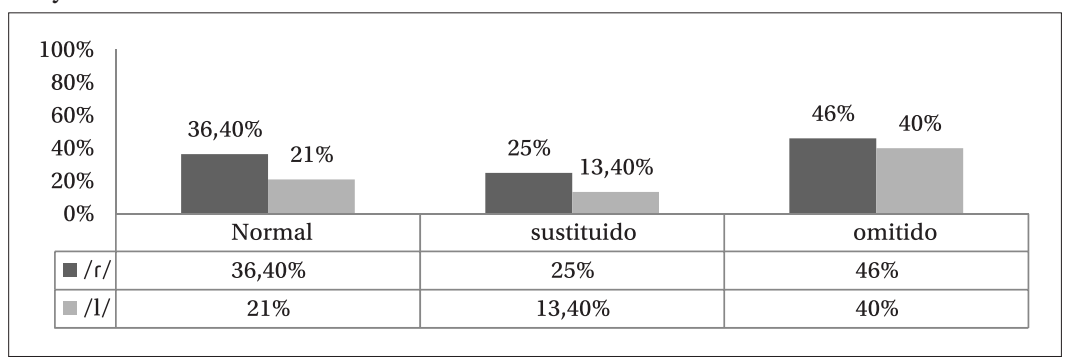


Por último, cabe señalar que estas diferencias en el porcentaje (y en el número) de emisiones infantiles correctas, sustituidas y omitidas para las codas consonánticas / / / y /l/ son estadísticamente significativas. En consecuencia, el porcentaje de emisiones correctas en la coda /l/ es un $15,5 \%$ más alto que en el caso de la coda $/ \mathrm{f} /$, mientras que en las sustituciones y omisiones evidencia que el porcentaje es un 11,5\% y un $6,1 \%$ menor en la coda / $/$ respecto de la coda / $/$, según lo presentado a continuación en la Tabla $\mathrm{N}^{\circ} 3$.

Tabla $\mathbf{N}^{\circ}$ 3. Diferencias de medias estadísticas en el porcentaje de producción de Codas / / / y /l/ según tipo de emisión (normal, sustituido y omitido).

\begin{tabular}{|c|c|c|c|c|c|c|c|}
\hline \multirow{2}{*}{\multicolumn{2}{|c|}{$\begin{array}{l}\text { Codas/ } \\
\text { Emisiones }\end{array}$}} & \multicolumn{2}{|c|}{ Normal (correcta) } & \multicolumn{2}{|c|}{ Sustituido } & \multicolumn{2}{|c|}{ Omitido } \\
\hline & & \multirow{2}{*}{ 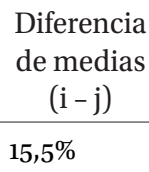 } & \multirow{2}{*}{$\begin{array}{l}\text { Sig. } \\
\text {,000* }\end{array}$} & \multirow{2}{*}{$\begin{array}{c}\text { Diferencia } \\
\text { de medias } \\
(\mathrm{i}-\mathrm{j})\end{array}$} & \multirow{2}{*}{$\begin{array}{l}\text { Sig. } \\
, 000^{*}\end{array}$} & \multirow{2}{*}{$\begin{array}{l}\text { Diferencia } \\
\text { de medias } \\
(i-j)\end{array}$} & \multirow{2}{*}{$\begin{array}{r}\text { Sig } \\
, 048^{*}\end{array}$} \\
\hline$/ \mathbf{l} /(\mathbf{i})$ & $/ \mathbf{f} /(\mathbf{j})$ & & & & & & \\
\hline
\end{tabular}

**Diferencias de medias significativas con valor-p $<0.05$. Test $\mathrm{t}$ - student de 2 grupos independientes/Test de U Mann-Whitney.

\subsection{Articulaciones correctas, sustituidas y omitidas según variable edad.}

En relación con la variable edad, se evidenció que tanto la articulación correcta de la coda líquida lateral /1/ como de la coda líquida vibrante $/ \mathrm{f} /$, aumentan según la edad de los participantes, a pesar de que los porcentajes difieren en ambas codas (Gráfico $\mathrm{N}^{\circ} 2$ ). En consecuencia, la coda / / se emite correctamente un 16,4\% en el I grupo etario (2,0-2,5 años), mientras que la coda $/ \mathrm{f} /$ se emite un $3,5 \%$ de forma correcta en este mismo grupo. Por otro lado, en el IV grupo de edad (3,6-3,11 años) la coda / $/$ / se emite un $52,1 \%$ de forma correcta; $y$, su contraparte, / $/$ / se emite correctamente tan solo un 36,2\%. Así, el porcentaje de articulación correcta de /l/ es mayor que el de / // según rango etario. 
Gráfico $\mathrm{N}^{\circ}$ 2. \% de emisiones correctas de las codas / / / y /l/ según rango etario.

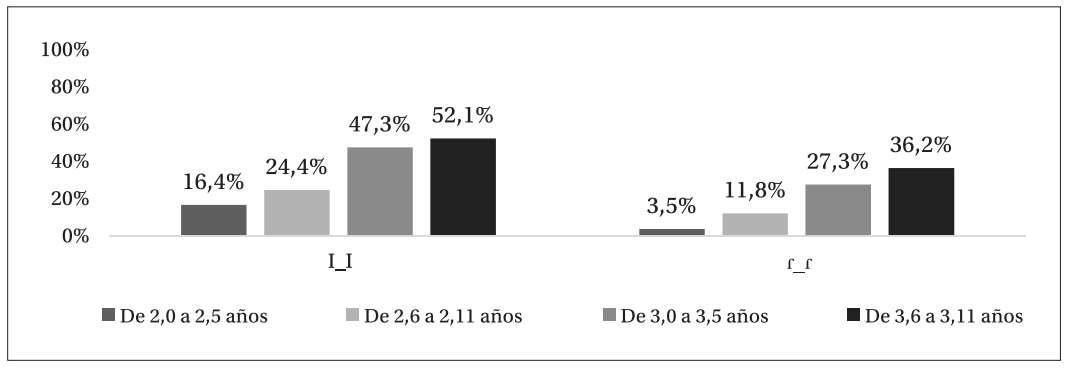

En la Tabla 4, se presenta el análisis de diferencia de medias según grupo de edad o tango etario para las codas consonánticas / f/ y/l/. Tal como se puede observar, se evidencia una diferencia estadísticamente significativa en la producción de emisiones correctas para ambas codas mencionadas, entre casi todos los pares de edad conformados. También cabe señalar que esta diferencia se incrementa a medida que aumenta la edad de los niños, existiendo una relación directamente proporcional entre el rango etario y el aumento del porcentaje de emisión correcta.

Tabla $\mathbf{N}^{\circ}$ 4. Diferencia media en el porcentaje de emisión correcta de Codas / / / y /l/ según rango etario.

\begin{tabular}{|c|c|c|c|c|c|c|}
\hline \multirow{2}{*}{$\begin{array}{l}\text { \% Emisiones } \\
\text { correctas }\end{array}$} & \multicolumn{3}{|c|}{$/ \mathrm{s} /$} & \multicolumn{3}{|c|}{ /1/ } \\
\hline & \multicolumn{3}{|c|}{ Diferencia de medias ( $\mathbf{i}$ - j) } & \multicolumn{3}{|c|}{ Diferencia de medias ( $\mathbf{i}$ - j) } \\
\hline \multirow{2}{*}{$\begin{array}{l}\text { G1: De 2,o a 2,5 } \\
\text { años (i) }\end{array}$} & G2 (j) & G3 (j) & $\mathrm{G}_{4}(\mathrm{j})$ & G2 (j) & G3 (j) & $\mathrm{G}_{4}(\mathrm{j})$ \\
\hline & $-8,00 \%$ & $-30,9 \% *$ & $-35,7 \%^{*}$ & $-8,4 \% *$ & $-23,8 \% *$ & $-32,7 \% *$ \\
\hline \multirow{2}{*}{$\begin{array}{l}\text { G2: De 2,6 a 2,11 } \\
\text { años (i) }\end{array}$} & $\mathrm{G} 1(\mathrm{j})$ & G3 (j) & $\mathrm{G}_{4}(\mathrm{j})$ & G1 (j) & G3 (j) & $\mathrm{G}_{4}(\mathrm{j})$ \\
\hline & $8,00 \%$ & $-22,9 \%^{*}$ & $-27,7 \% *$ & $8,4 \% *$ & $-15,4 \% *$ & $-24,4 \% *$ \\
\hline \multirow{2}{*}{$\begin{array}{l}\text { G3: De 3,o a 3,5 } \\
\text { años (i) }\end{array}$} & $\mathrm{G} 1(\mathrm{j})$ & G2 (J) & $\mathrm{G}_{4}(\mathrm{j})$ & $\mathrm{G} 1(\mathrm{j})$ & G2 (j) & $\mathrm{G}_{4}(\mathrm{j})$ \\
\hline & $30,9 \% *$ & $22,9 \%^{*}$ & $-4,80 \%$ & $23,8 \% *$ & $15,4 \%^{*}$ & $-8,90 \%$ \\
\hline \multirow{2}{*}{$\begin{array}{l}\text { G4: De } 3,6 \text { a 3,11 } \\
\text { años (i) }\end{array}$} & $\mathrm{G} 1(\mathrm{j})$ & G2 (j) & G3 (j) & $\mathrm{G} 1(\mathrm{j})$ & G2 (j) & G3 $(j)$ \\
\hline & $35,7 \% *$ & $27,7 \% *$ & $4,80 \%$ & $32,7 \% *$ & $24,4 \% *$ & $8,90 \%$ \\
\hline
\end{tabular}

*Diferencias de medias significativas con valor-p $<0.05$. Test Anova de un factor/Test de Kruskal Wallis.

En cuanto al fenómeno articulatorio de sustitución presentado en el Gráfico $\mathrm{N}^{\circ} 3$, se evidenció que este es más frecuente en la coda líquida vibrante / $/$ / que en la coda líquida lateral /l/ en todos los grupos de edad 
estudiados. Sin embargo, cabe señalar que el porcentaje de sustitución de la coda / $\mathrm{s}$ / se mantiene similar en los tres primeros grupos etarios, presentándose de la siguiente forma: $23,4 \%$ (2,0-2,5 años), 23,3\% (2,62,11 años) y $23,1 \%(3,0-3,5)$. En el caso del IV grupo etario $(3,6-3,11$ años) el porcentaje de sustitución aumenta con una diferencia de alrededor de $6 \%$ respecto del III grupo de edad $(23,1 \%)$. Este porcentaje de sustitución de la coda / / / entre el rango etario de 3,6-3,11 años es de un $29,6 \%$.

En cuanto al porcentaje de sustitución de la coda /1/, se observa que esta también se mantiene similar en los tres primeros grupos etarios, siendo de la siguiente forma: $10,9 \%$ (2,0-2,5 años), 11,2\% (2,6-2,11 años) y 12,3\% (3,0-3,5 años). Pero al igual que con la sustitución de la coda / $/$ / este porcentaje se incrementa en el IV grupo etario (3,6-3,11 años), en un $6,3 \%$ respecto del grupo etario III (3,0 a 3,5 años). En consecuencia, como primera observación, a nivel descriptivo no se observa que el porcentaje de sustitución de las codas analizadas aumente o disminuya en cada uno de los grupos de edad analizados.

Gráfico $\mathrm{N}^{\circ}$ 3. \% de sustituciones de las codas / / / y /l/ según rango etario.

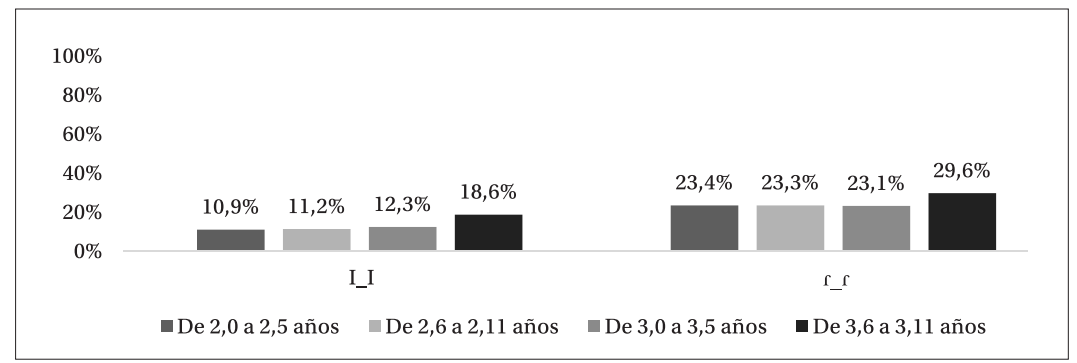

En la Tabla 5, se presenta el análisis de diferencia de medias de sustituciones según grupo de edad o rango etario para las codas

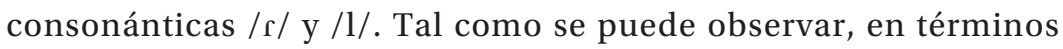
inferenciales, no se evidencian diferencias estadísticamente significativas en los pares de edad conformados para cada una de las codas descritas, pese a las diferencias descritas en los párrafos anteriores. De este modo, se evidencia que la sustitución no aumenta ni disminuye con la edad; o, dicho de otra manera: la edad no está relacionada con los niveles porcentuales de sustitución para las codas /s/ y /1/, respectivamente. 
Tabla $\mathbf{N}^{\circ}$. Diferencias de medias en el porcentaje de sustituciones de Codas / $/$ / y /1/ según rango etario.

\begin{tabular}{|c|c|c|c|c|c|c|}
\hline \multirow{2}{*}{ \% Sustituciones } & \multicolumn{3}{|c|}{$/ \mathrm{s} /$} & \multicolumn{3}{|c|}{$/ 1 /$} \\
\hline & \multicolumn{3}{|c|}{ Diferencia de medias ( $\mathrm{i}-\mathrm{j}$ ) } & \multicolumn{3}{|c|}{ Diferencia de medias ( $\mathrm{i}-\mathrm{j}$ ) } \\
\hline \multirow{2}{*}{$\begin{array}{l}\text { G1: De 2,o a 2,5 } \\
\text { años (i) }\end{array}$} & G2 (j) & G3 (j) & $\mathrm{G}_{4}(\mathrm{j})$ & G2 (j) & G3 (j) & $\mathrm{G}_{4}(\mathrm{j})$ \\
\hline & $-0,30 \%$ & $-1,40 \%$ & $-7,70 \%$ & $0,00 \%$ & $0,30 \%$ & $-6,20 \%$ \\
\hline \multirow{2}{*}{$\begin{array}{l}\text { G2: De 2,6 a 2,11 } \\
\text { años (i) }\end{array}$} & $\mathrm{G} 1(\mathrm{j})$ & G3 (j) & $\mathrm{G}_{4}(\mathrm{j})$ & $\mathrm{G} 1(\mathrm{j})$ & G3 (j) & $\mathrm{G}_{4}(\mathrm{j})$ \\
\hline & $0,30 \%$ & $-1,10 \%$ & $-7,40 \%$ & $0,00 \%$ & $0,30 \%$ & $-6,20 \%$ \\
\hline \multirow{2}{*}{$\begin{array}{l}\text { G3: De } 3,0 \text { a 3,5 } \\
\text { años (i) }\end{array}$} & $\mathrm{G} 1(\mathrm{j})$ & G2 (J) & $\mathrm{G}_{4}(\mathrm{j})$ & $\mathrm{Gl}(\mathrm{j})$ & G2 (j) & $\mathrm{G}_{4}(\mathrm{j})$ \\
\hline & $1,40 \%$ & $1,10 \%$ & $-6,30 \%$ & $-0,30 \%$ & $-0,30 \%$ & $-6,50 \%$ \\
\hline \multirow{2}{*}{$\begin{array}{l}\text { G4: De } 3,6 \text { a 3,11 } \\
\text { años (i) }\end{array}$} & $\mathrm{Gl}(\mathrm{j})$ & G2 (j) & G3 $(j)$ & $\mathrm{G} 1(\mathrm{j})$ & G2 (j) & $\mathrm{G}_{3}(\mathrm{j})$ \\
\hline & $7,70 \%$ & $7,40 \%$ & $6,30 \%$ & $6,20 \%$ & $6,20 \%$ & $6,50 \%$ \\
\hline
\end{tabular}

*No se evidencian diferencias de medias significativas con valor-p $<0.05$. Test Anova de un factor/Test de Kruskal Wallis.

Finalmente, en relación con el fenómeno de omisión, se evidenció que esta en las codas / $/$ y / / disminuye a medida que avanza la edad, aunque con un leve incremento en el rango etario de 2,6 a 2,11 años para ambas codas señaladas. De este modo, mientras que en el I grupo etario de 2,0 a 2,5 años la coda /1/ se omite un $49,1 \%$ y la coda / $/$ / se omite un 50,2\%; entre los 3,6-3,11 años (IV grupo etario) el porcentaje de omisión de la coda / / y la coda / $/$ / disminuye a un $26 \%$ y $31,6 \%$, según lo que se presenta en el Gráfico $\mathrm{N}^{\circ} 4$.

Gráfico $\mathbf{N}^{\circ}$ 4. \% de omisiones de las codas /§/ y /l/ según rango etario.

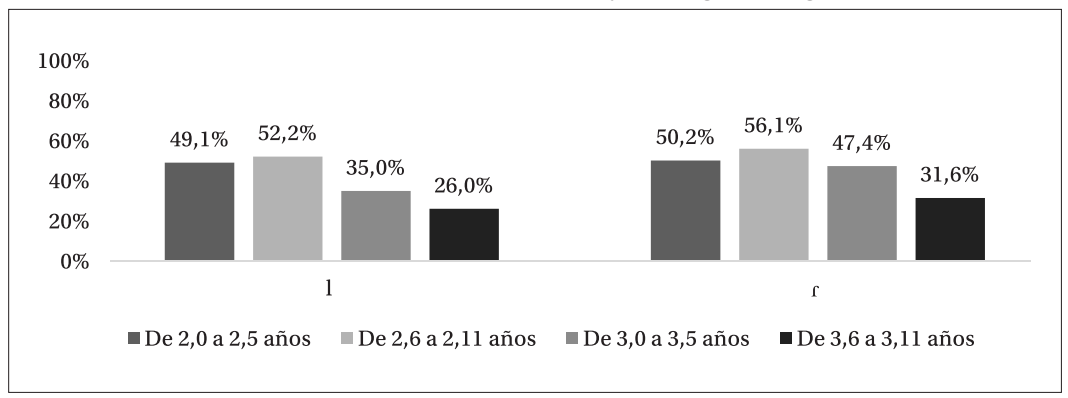

Como se puede observar, el porcentaje de omisión en ambas codas disminuye a medida que se incrementa la edad, siendo más notoria esta diferencia en los grupos etarios III y IV respecto de grupos I y II. En 
la Tabla $\mathrm{N}^{\circ} 6$ se presenta que estas son estadísticamente significativas con un valor-p inferior a 0,05 ; es decir, que las diferencias observadas corresponden a una disminución efectivamente existente entre los grupos etarios con respecto del porcentaje de omisión de ambas codas.

Tabla $\mathbf{N}^{\circ}$ 6. Diferencias de medias en el porcentaje de omisiones de Codas / $/ \mathrm{y}$ /l/ según rango etario.

\begin{tabular}{|c|c|c|c|c|c|c|}
\hline \multirow{2}{*}{ \% Omisiones } & \multicolumn{3}{|c|}{$/ \mathrm{s} /$} & \multicolumn{3}{|c|}{$/ \mathrm{l} /$} \\
\hline & \multicolumn{3}{|c|}{ Diferencia de medias ( $\mathrm{i}$ - j) } & \multicolumn{3}{|c|}{ Diferencia de medias ( $\mathrm{i}-\mathrm{j}$ ) } \\
\hline \multirow{2}{*}{$\begin{array}{l}\text { G1: De 2,0 a 2,5 } \\
\text { años (i) }\end{array}$} & $\mathrm{G} 2(\mathrm{j})$ & G3 (j) & G4 (j) & G2 (j) & G3 (j) & $\mathrm{G}_{4}(\mathrm{j})$ \\
\hline & $-3,1 \%$ & $14,1 \%$ & $23 \% *$ & $-5,9 \%$ & $2,8 \%$ & $18,7 \% *$ \\
\hline \multirow{2}{*}{$\begin{array}{l}\text { G2: De 2,6 a 2,11 } \\
\text { años (i) }\end{array}$} & $\mathrm{G} 1(\mathrm{j})$ & G3 (j) & $\mathrm{G}_{4}(\mathrm{j})$ & $\mathrm{G} 1(\mathrm{j})$ & G3 $(j)$ & $\mathrm{G}_{4}(\mathrm{j})$ \\
\hline & $3,1 \%$ & $17,2 \% *$ & $26,1 \% *$ & $5,9 \%$ & $8,7 \%$ & $24,5 \% *$ \\
\hline \multirow{2}{*}{$\begin{array}{l}\text { G3: De 3,0 a 3,5 } \\
\text { años (i) }\end{array}$} & $\mathrm{G} 1(\mathrm{j})$ & G2 (J) & $\mathrm{G}_{4}(\mathrm{j})$ & $\mathrm{G} 1(\mathrm{j})$ & G2 (j) & $\mathrm{G}_{4}(\mathrm{j})$ \\
\hline & $-14,1 \%$ & $-17,2 \% *$ & $9,0 \%$ & $-2,8 \%$ & $-8,7 \%$ & $15,8 \% *$ \\
\hline \multirow{2}{*}{$\begin{array}{l}\text { G4: De } 3,6 \text { a 3,11 } \\
\text { años (i) }\end{array}$} & $\mathrm{G} 1(\mathrm{j})$ & G2 (j) & G3 (j) & G1 (j) & G2 (j) & G3 $(j)$ \\
\hline & $-23 \% *$ & $-26,1 \% *$ & $-9,0 \%$ & $-18,7 \%^{*}$ & $-24,5 \% *$ & $-15,8 \% *$ \\
\hline
\end{tabular}

*Diferencias de medias significativas con valor-p $<0.05$. Test Anova de un factor/ Test de Kruskal-Wallis.

\subsection{Tipos de sustituciones para la coda líquida vibrante / / /}

Como ya se ha señalado en el apartado metodología (Sección 3) la coda líquida vibrante / $\mathrm{f}$ / se evaluó en las palabras árbol, cárcel, torta, enfermo. En términos generales, la coda fue sustituida por los segmentos [1], [n], [ð], [h], [t], [j], [a] y [w]; mientras que en cuanto a la frecuencia de sustituciones la coda / / se sustituyó más veces por la líquida lateral /l/ (47\%), seguida por la nasal /n/ (13\%) y en último lugar por la /w/ (3\%). Algunos ejemplos de sustituciones de la coda /r/ por los segmentos /l/, /n/ y /ð/ son los siguientes: a) "árbol”; ['al.ßol] (2,9 años), "cárcel”: ['kal.sel] (2,9 años), "torta": ['tol.ta] (2,9 años), "enfermo": ['fel.mo] (3,o años); b) "torta": ['ton.ta] (3,3 años) y; c) "torta": ['toð.ta] (3,4 años). Otras sustituciones menos frecuentes fueron, por ejemplo: "torta": [ tohta] (3,0 años), "torta": ['toj.ta] (2,5 años), "cárcel": ['kat.sel] (2,9 años). 
Gráfico $\mathbf{N}^{\circ}$ 5. \% y tipos de sustitución de la coda / / /

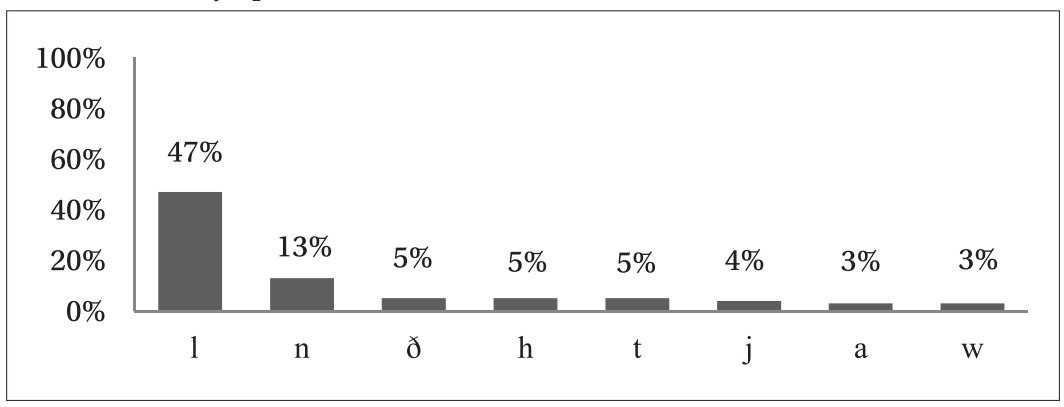

\subsection{Tipos de sustituciones para la coda líquida lateral ///}

La coda líquida lateral se evaluó en las láminas bolsa, alto, falda. Las sustituciones más frecuentes de esta coda fueron por los segmentos: [r], $[\mathrm{n}],[\mathrm{j}],[\mathrm{t}],[\mathrm{h}],[\mathrm{k}],[\mathrm{s}] \mathrm{y}[\mathrm{\partial}]$; mientras que, en cuanto a la frecuencia de sustituciones la coda /l/ se sustituyó más veces por la líquida vibrante / $/$ (26\%), seguida por la nasal /n/ (23\%\%) y en último lugar por /ð/ (3\%).

Gráfico $\mathbf{N}^{\circ}$ 6. \% y tipos de sustitución de la coda /1/.

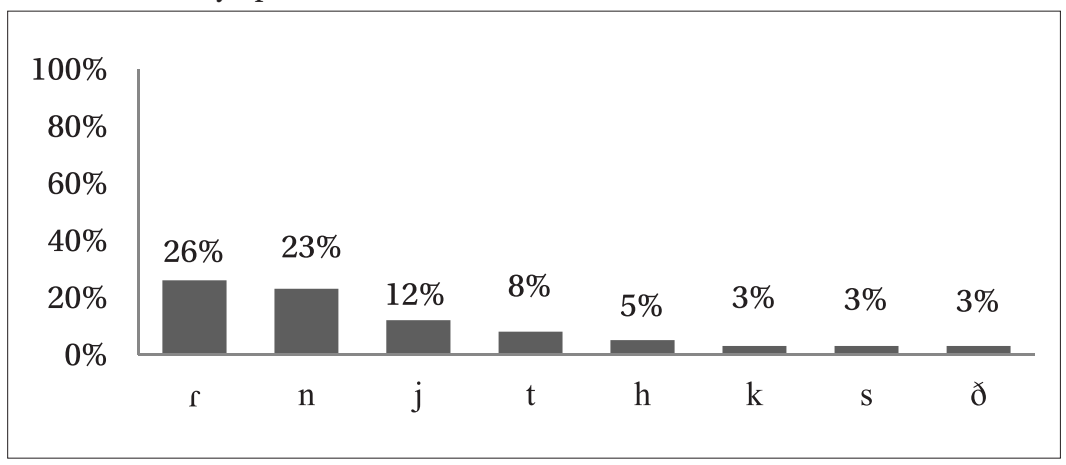

Algunos ejemplos de sustituciones de la coda /l/ por los segmentos /r/, /n/y /j/ son los siguientes: a) "bolsa": ['bor.sa] (3,6 años), "alto": ['ar. to] (3,0 años); b) "alto": ['dzan.to] (3,7 años), "falda": ['fan.da] (2,8 años) y; c) "alto": ['aj.to] (2,2 años), "falda": ['fajda] (3,3 años). Otras sustituciones menos frecuentes fueron, por ejemplo: "alto" [ aðto] (3,11 años), "alto": ['akto] (2,5 años), ['ah.to] (3,6 años). 


\section{Conclusiones}

\section{Rango etario}

Una de las ventajas de esta investigación fue poder analizar la adquisición de las codas consonánticas antes de los 3 años, lo cual se considera como la edad apropiada para describir el desarrollo de la fonología infantil. Tal como era esperable, se evidenció que tanto la articulación correcta de la coda lateral /l/ como de la coda vibrante / $/$ /, aumentan según la edad de los participantes, a pesar de que los porcentajes difieren en ambas codas. En definitiva, alrededor de los tres años de edad, el 50\% de los niños analizados en la muestran articulan la coda medial /1/; mientras que, en el caso de la coda / $/$ / el porcentaje no llega al $40 \%$. Los resultados anteriores, indican que, estas estructuras corresponden a adquisiciones tardías, a pesar de que la coda es una estructura que emerge a muy temprana edad, específicamente alrededor del año (Morales-Front, 2006). Los resultados de esta investigación coinciden con los de Diez-Itza \& Martínez (2004) quienes analizaron la producción de los grupos heterosilábicos desde la perspectiva de los procesos fonológicos de simplificación en 240 niños hablantes de español desde los 3,0 a los 5,11 años de edad. En su estudio establecieron tres etapas: 1) expansión, 2) estabilización; 3) resolución. Según los autores, la reducción de PFS de estas estructuras presentan un perfil de decremento de PFS muy gradual y que aparece muy tendido durante la etapa de estabilización que ocurriría, según las autoras, luego de los 3,6 años.

\section{Adquisición de codas mediales según tipo de segmento}

Se evidenció que la articulación correcta de la coda /1/ es mayor que la de /r/ (36,4\% vs. 21\%, respectivamente). Los resultados anteriores, coinciden con la mayoría de la literatura que ha evidenciado que las primeras codas mediales en emerger son las nasales y laterales, mientras que, las últimas en ser adquiridas serían las vibrantes (Fernández López, 2009; Goldstein \& Cintrón, 2001; Núñez-Cedeño, 2007, Polo, 2018). Como ya se ha mencionado en el marco teórico entre los variados factores que intervienen en la adquisición de las codas, uno tiene que ver con los rasgos propios del segmento que ocupa la coda. Por ejemplo, Bosch (2004) observó un patrón evolutivo que comienza con la realización de las codas nasales y laterales, y termina con las fricativas y vibrantes. La autora señala que 
estas últimas todavía pueden estar sujetas a errores de producción a los 6 años, en especial en el caso de la vibrante [- $[$ ], con un interesante paralelismo entre esta dificultad y la que se observa en la realización de la vibrante múltiple en posición de ataque silábico y coda. La importancia de distinguir ente los aspectos prosódicos y los articulatorios durante la adquisición de los grupos consonánticos en general es destacado por Oropeza Escobar (2001) quien distingue entre las restricciones posicionales y las restricciones intersegmentales. Las restricciones intersegmentales tienen que ver con las características fonéticas de los segmentos que participan en una secuencia, con bastante libertad respecto a su localización; mientras que, las restricciones "posicionales" obedecen a la ubicación del segmento con relación a un cierto linde, es decir, a una determinada unidad lingüística.

\section{Procesos de sustitución y omisión en la adquisición de las codas mediales}

En cuanto al fenómeno articulatorio de sustitución se evidenció que este es más frecuente en la coda líquida vibrante / $/$ que en la coda líquida lateral /1/ en todos los grupos de edad estudiados; mientras que, en el casi de la omisión el porcentaje se mantiene similar en ambas tipos de codas. En cuanto a la variable edad, no se observa que el fenómeno de sustitución disminuya significativamente con el aumento de la edad de los participantes, sino que más bien, se evidencia un período de estancamiento o como denomina Diez-Itza \& Martínez (2004) una etapa de "estabilización" de los PFS en el que su desaparición es muy gradual. A diferencia del fenómeno anterior, en el caso de la omisión se observó que este disminuye a medida que avanza la edad en ambos tipos de codas. En relación con la frecuencia de aparición entre la sustitución y la omisión se observó que la omisión es mucho más frecuente que la sustitución en todos los grupos etarios analizados. El fenómeno anterior se evidencia también en el estudio de Polo (2018).

\section{Sobre los tipos de sustitución}

En términos generales, la coda / $/$ fue sustituida con más frecuencia por los segmentos [l], [n], [ð], [h], [t], [j], [a] y [w]; mientras que, las sustituciones más frecuentes para la coda /l/ fueron por los segmentos: [r], [n], $[\mathrm{j}],[\mathrm{t}],[\mathrm{h}],[\mathrm{k}],[\mathrm{s}]$ y $[ð]$. Si en primer lugar, se comparan este tipo de sustituciones con las que los niños suelen producir para /l/ y / / / en posición 
aislada se encuentran evidentes similitudes. Por ejemplo, para el caso de la vibrante simple, variados estudios para el español han evidenciado que este sonido es frecuentemente sustituido por la lateral [1] y la fricativa [ð] (Anderson \& Smith, 1987; Bosch, 2004, Vivar, 2009); mientras que, además otros autores afirman que al comienzo de su adquisición del español los niños sustituyen las codas por semiconsonantes ([w, j]) (Borràs-Comes \& Prieto, 2013; Lleó et al. , 2003; Morales-Front, 2006; Oropeza Escobar, 2001). En segundo lugar y, con respecto al punto de articulación, se evidencia en los resultados que en ambos tipos de codas los niños tienden a mantener el punto de articulación alveolar al momento de sustituir la coda. En definitiva, durante la adquisición de las codas líquidas mediales se evidenciaron patrones de sustitución de las mismas que se relacionan con las características articulatorias de estos segmentos. Sobre los tipos de sustitución de estas codas, Polo (2018) en su estudio observó que las codas sonorantes ${ }^{2}$ (es decir, vibrantes y laterales) se sustituyen con un sonorante. Este mismo patrón de sustitución se evidencia en el estudio actual. Según la autora, este es un hallazgo importante porque puede ofrecer una explicación para los patrones de sustitución de los niños: muestra que las sustituciones están ordenadas, y que los niños sustituyen los sonidos por otros sonidos que pertenecen a la misma clase de sonidos que están siendo reemplazados.

\section{Referencias bibliográficas}

Anderson, R. \& Smith, B. (1987). Phonological development of two-yearold monolingual Puerto Rican Spanish-speaking children. Journal of Child Language, 14(1), 57-78.

Bosch, L. (2004). Evaluación fonológica del habla infantil. Barcelona: Elsevier Masson.

Borràs-Comes, J. \& Prieto, P. (2013). The acquisition of coda consonants by Catalan and Spanish children: Effects of prominence and frequency of exposure. Probus, 26(1), 59-82.

Clements, G. N. (1990). The role of the sonority cycle in core syllabification. In J. Kingston, \& M. E. Beckman (Eds.), Papers in laborato- 
ry phonology I. Between the grammar and the physics of speech, (pp. 283-333). Cambridge, UK: Cambridge University Press.

Diez-Itza, E., Martínez, V. (2004). Las etapas tardías de la adquisición fonológica: procesos de reducción de grupos consonánticos. Anuario de Psicología, 2(35), 177-202. Facultat de Psicologia Universitat de Barcelona.

Fernández López, I. (2009). ¿Cómo hablan los niños? El desarrollo del componente fonológico en el lenguaje infantil [How do children speak? Development of the phonological component in children's language]. Madrid, Spain: Arco/Libros.

Fey, M. E., \& Gandour, J. (1982). The pig dialogue: Phonological systems in transition. Journal of Child Language, 9(2), 517-519.

Fikkert, P. (1994). On the acquisition of prosodic structure. Holland Academic Graphics: The Hague.

Freitas, M. J. (1997). Aquisição da Estrutura Silábica do Português Europeu. (Tesis Doctoral). University of Lisbon, Lisboa, Portugal.

Goldstein, B., \& Cintrón, P. (2001). An investigation of phonological skills in Puerto-Rican Spanish-speaking 2-year-olds. Clinical Linguistics and Phonetics, 15, 343-361.

Goad, H., \& Brannen, K. (2003). Phonetic evidence for phonological structure in syllabification. En J. Van de Weijer, V. van Heuven, H. van der Hulst (Eds.), The phonological spectrum (pp. 3-30). Amsterdam: John Benjamins.

Harris, J. (1991). La estructura silábica y el acento en español: Análisis no lineal. (Trad. de O. Soriano). Visor: Madrid.

Jusczyk, P. (1997). The discovery of spoken language. MA: MIT Press: Cambridge.

Kehoe, M. \& Stoel- Gammon, C. (2001). Development of syllable structure in English-speaking children with particular reference to rhymes. Journal of Child Language, (28), 393- 432.

Kirk, C. (2008). Substitution errors in the production of word-initial and word-final consonant clusters. Journal of Speech, Language, and Hearing Research, 51, 1-14.

Kirk, C. \& Demuth, K. (2006). Accounting for variability in 2-year-olds' production of coda consonants. Language Learning and Development, 2, 97-118. 
Lleó, C. (2003). Prosodic licensing of codas in the acquisition of Spanish. Probus, 15, 257-281.

Lleó, C., Kuchenbrandt, I., Kehoe, M., \& Trujillo, C. (2003). Syllable final consonants in Spanish and German monolingual and bilingual acquisition. In N. Müller (Ed.), (In)vulnerable domains in multilingualism (pp.191-220). Hamburg, Germany: University of Hamburg.

MacNeilage, P.F. \& Davis, B.L. (1990). Acquisition of speech production: Achievement of segmental independence. Hardcastle, W.I. \& Marchal A. (Eds.) Speech Production and Speech Modeling (pp. 55-68). Dordrecht: Kluwer.

Morales-Front, A. (2006). Acquisition of syllable structure in Spanish. In F. Martínez Gil, \& S. Colina (Eds.), Optimality-theoretic studies in Spanish phonology (pp. 500-524). Amsterdam, The Netherlands: John Benjamins.

Núñez-Cedeño, R. (2007). The acquisition of Spanish codas: A frequency/ sonority approach. Hispania, 90, 147-163.

Oropeza Escobar, M. (1987). El curso del desarrollo consonántico en niños hispanohablantes de 2 a 6 años de edad. (Tesis de licenciatura). Facultad de Antropología, Universidad Veracruzana, Xalapa, México.

(2001). Adquisición de la estructura silábica del español en niños de dos a seis años. Colección Pedagógica Universitaria, 36, 1-13.

Polo, N. (2018). Acquisition of codas in Spanish as a first language: The role of accuracy, markedness and frequency. First Language, vol. 38(1), 3-25.

Prieto, P. (2006). The relevance of metrical information in early prosodic word acquisition: A comparison of Catalan and Spanish. Language and Speech, 49, 231-259.

Prieto, P., \& Bosch-Baliarda, M. (2006). The development of codas in Catalan. A. Gavarró, C. Lleó (Eds.). Catalan Journal of Linguistics, 5(Special issue on L1 Acquisition of Romance), 237-272.

Quilis, A. (1999). Tratado de fonética y fonología españolas. Gredos, Biblioteca Románica: Madrid.

Rose, Y. (2000). Place specification and segmental distribution in the acquisition of word final consonant syllabification. The Canadian 
Journal of Linguistics / La Revue Canadienne de Linguistique, 48, 409-435.

Stoel, C. (1974). Note on the acquisition of sonorants in Spanish. Papers and reports on child language development. Palo Alto, CA: Stanford University.

Song, J.Y, Demuth. K., \& Shattuck-Hufnagel, S. (2012). The development of acoustic cues to coda contrasts in young children learning American English. The Journal of the Acoustical Society of America, 131(4), 36-50.

Stites, J., Demuth, K., \& Kirk, C. (2004). Markedness vs. Frequency Effects in Coda Acquisition. In Alejna Brugos, Linnea Micciulla, and Christine E. Smith (Eds), Proceedings of the 28th Annual Boston University Conference on Language Development (pp. 565-76). Somerville, MA: Cascadilla Press.

Stoel-Gammon, C. (1998). Sounds and words in early language acquisition: the relationship between lexical and phonological development. In R. Paul (Ed.), Exploring the speechlanguage connection. Baltimore: Paul H. Brookes Publishing Co.

Vivar, P. \& León, H. (2009). Desarrollo fonológico-fonético en un grupo de niños entre 3 y 5,11 años. CEFAC, 2(11), 190-198.

Vivar, P. (2009). Evaluación de grupos consonánticos de ataque complejo en un grupo de niños de la ciudad de Concepción con prueba articulatoria CEFI. Onomazéin, 20(2), 33-44.

Zamuner, T., Gerken, L., \& Hammond, M. (2004). Phonotactic probabilities in young children's speech production. Journal of Child Language, 3(31), 515 - 536. 